pass such a testing regime.) This violates a cardinal principle of regulation: that the degree of regulatory scrutiny should be commensurate with risk.

Like many other regulatory approaches that are limited to recombinant DNA-derived products, the $\mathrm{FAO} / \mathrm{WHO}$ allergenicity protocol is compromised by adopting a scope that makes no scientific sense. The use of recombinant DNA technology—a stunning refinement of older, less precise techniques-generates less uncertainty about possible untoward effects. But for recombinant organisms, and the foods derived from them, both the fact and degree of regulation are determined by the production methods. That makes no sense.

Henry I. Miller

Hoover InstitutionStanford,

CA 94305

(miller@hoover.stanford.edu) and Gregory Conko

Competitive Enterprise Institute Washington, DC 20036

(conko@cei.org)

\section{The production capacity bottleneck}

\section{To the editor:}

The article by Ken Garber in your March issue (Nat. Biotechnol. 19, 186-187, 2001), raises the issue of the production capacity challenge that faces the biotechnology industry and threatens to increase the time and cost of development. The fact that a company such as Abgenix announced in February that it has booked a good manufacturing practice (GMP) suite at a Lonza facility for 5 years is just a confirmation of this bottleneck.

Increasing the existing cell culture capacity is one obvi-

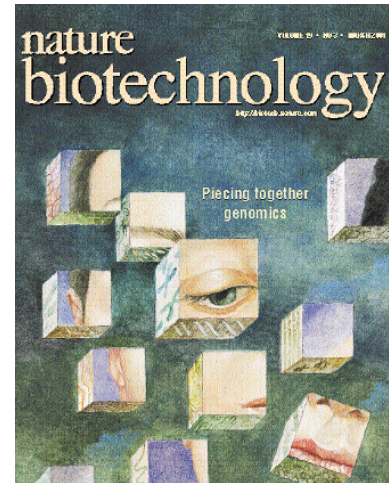

candidates for development and pushing them as fast as possible to the market. Developing new cell culture or purification system is not a priority in this scheme. Reaching "acceptable" standard productivity is the target for the development team because they have to work with standard production processes.

Ken Garber identifies two approaches to increase the productivity, either by increasing the expression levels or improving protein recovery. A third approach is also available in which cell culture technology can be combined with downstream processing.

Using perfused $60 \mathrm{~L}$ bioreactors instead of the standard batch process, more than tenfold increases in culture densities can be obtained. In addition, the perfused medium flowing out of a high-density perfused bioreactor can be directly processed in expanded bed mode chromatographic systems without any further filtration, clarification, or concentration steps. Protein harvesting carried out on a day-byday basis concentrates protein ready for later pooling and downstream processing.

This process has the advantage of being amenable to implementation using existing bioreactors, reduces costs of production, and addresses production capacity by putting 10-20-fold more cells "to work" in the existing bioreactor. In addition, by relying on the $60 \mathrm{~L}$ bioreactor (a mobile low cost unit), material for clinical studies up to phase III reduces dramatically the investment required to prove the efficacy of the hundreds of molecules reported to be in development. Large-capacity bioreactors can then be freed for the production of commercial batches.

$$
\begin{array}{r}
\text { Jean-Luc De Keyser } \\
4 \text { C Biotech } \\
14 \text { rue de la Marlette } \\
7180 \text { Seneffe } \\
\text { Belgium } \\
\text { (jl.dekeyser@4c.be) }
\end{array}
$$

Surprisingly enough, very few improvements have been brought to culture processes so far. Most industrial-scale biotechnological processes rely on similar technologies to those used for laboratory-scale operation. This is quite unique in the industrial world. In a rather provocative way, one could say that cell culture remains a matter of agitating and aerating culture medium in tanks and obtaining the required quantities by "simply" scaling up the equipment.

Reasons for this are numerous. One very relevant observation is that within large biotechnology companies, the pressure is on the identification of new target or molecules

\section{Quality control of plasmid preparations}

To the editor:

In the mid-1970s, two simple methods were developed to determine gene sequence ${ }^{1,2}$. A common feature of these methods is that they require the subcloning of the DNA fragment of interest, for instance into a plasmid and its amplification in Escherichia coli. Assuming a rate of spontaneous mutation of $10^{-9}$, this amplification step leads to the presence of several thousand mutated plasmids in $1 \mu \mathrm{g}$ of plasmid DNA. However, these point mutations do not affect the accuracy of the determined gene sequence as a base substitution occurring at the same position in less than $15 \%$ of the population used as template is not detected by currently practiced DNA sequencing methods. This situation fulfils the aim of DNA sequencing, which is to determine the original sequence of a gene and not to detect the mutations occurring in this gene during bacterial amplification.

Nowadays, DNA sequencing is also used as a mean for quality control of plasmid preparations intended for clinical applications (i.e., gene therapy or DNA vaccination). In this case, the aim is not to determine the nucleotide sequence of the original plasmid used to transform bacteria, but to control the quality of a plasmid population obtained after amplification. However, as mentioned above, one of the important features of DNA sequencing methods is that they do not detect the quantitatively minor base substitutions occurring during plasmid amplification. Thus, this leads to the paradoxical situation in which DNA sequencing methods whose characteristic is not to detect base substitutions occurring during plasmid amplification are used precisely to certify the absence of such mutations in plasmid DNA populations obtained after amplification.

The resulting confusion may have dramatic consequences. Even if the high number of mutated plasmids corresponds to a very small percentage of the plasmid population, it may be possible for some mutations to give rise to noxious protein by a gain of a dominant aberrant function, as reported for instance for the tumor suppressor $p 53$. Thus, the control of plasmid DNA sequences intended for clinical use should involve more sensitive methods either specially designed for this aim or able to eliminate mutated DNA. Without such technological improvements, there is a risk that the use of therapeutic DNA for gene therapy or DNA vaccination could lead to the injection of numerous undetectable mutated plasmids with potentially unknown biological properties.

Laurence Gluck, Ludovic Alazay, David Ratel, Stéphanie Puget and Didier Wion.

INSERM U318,

CHU Michallon, 38043 Grenoble Cedex 09,

France

(Didier.wion@ujf-grenoble.fr)

1. Maxam, A.M. \& Gilbert, W. Proc. Natl. Acad. Sci. USA. 74, 560-564 (1977).

2. Sanger F, Nicklen S, \& Coulson AR. Proc. Natl. Acad. Sci. USA. 74, 5463-5467 (1977). 